\title{
Fatores de Mau Prognóstico nas Peças Operatórias de Pacientes Submetidos ao Tratamento Cirúrgico do Câncer Colorretal
}

\author{
Bad Prognostic Factors on the Operatory Peace of Patients Submitted to \\ Surgical Treatment of Colorectal Cancer
}

\author{
MARÍLIA GRANZOTTO VOLPATO' ${ }^{1}$, KAISER DE SOUZA KOCH ${ }^{2}$ \\ ${ }^{1}$ Acadêmica do $5^{\circ}$ ano do curso de Medicina da Universidade do Sul de Santa Catarina (UNISUL); \\ ${ }^{2}$ Professor orientador, responsável pela Disciplina de Cirurgia do Aparelho Digestivo da UNISUL, Mestre em Ciências \\ Médicas pela Universidade Federal de Santa Catarina.
}

\begin{abstract}
VOLPATO MG; KOCH KS. Fatores de Mau Prognóstico nas Peças Operatórias de Pacientes Submetidos ao Tratamento Cirúrgico do Câncer Colorretal. Rev bras Coloproct, 2008;28(4): 414-424.

RESUMO: INTRODUÇÃO: O estadiamento anátomo-patológico da peça operatória representa a principal ferramenta de aferição do prognóstico e sobrevida de pacientes com câncer colorretal (CCR). OBJETIVOS: Determinar a prevalência do T, N, grau de diferenciação celular e presença de mucina na peça operatória de pacientes submetidos ao tratamento cirúrgico do CCR e suas correlações. MÉTODOS: Os laudos anátomo-patológicos de 144 pacientes foram avaliados quanto ao estadiamento clínicopatológico. RESULTADOS: A média de idade encontrada foi de 61,21 anos. Doze pacientes tinham menos de 40 anos $(8,3 \%)$. Cento e vinte e cinco pacientes $(\mathbf{8 6 , 8 \%})$ apresentaram tumores grandes $(>35 \mathrm{~mm})$. Cento e doze pacientes $(\mathbf{7 7 , 8 \%})$ encontravamse no estádio $\mathrm{T} 3$ e 77 pacientes $(53,5 \%)$ foram classificados como N0. Setenta e seis pacientes $(52,8 \%)$ apresentaram adenocarcinoma bem diferenciado. Seis pacientes $(\mathbf{4 , 2} \%)$ tiveram tumores secretores de mucina. A média de gânglios dissecados foi de 11,1 e a média de gânglios positivos foi de 2,79. CONCLUSÃO: Pacientes jovens não apresentaram estadiamento T/N mais avançado ou maior indiferenciação celular/secreção de mucina. Tumores grandes obtiveram estádio $T$ avançado, sendo que os estadiamentos T e N correlacionaram-se positivamente com maior média de gânglios dissecados. A ressecção de maior número de linfonodos aumentou a chance de se encontrar gânglio neoplásico, e quanto maior o número de gânglios positivos, mais avançado foi o estadiamento T, N e o grau de indiferenciação celular/presença de mucina.
\end{abstract}

Descritores: Câncer colorretal, prognóstico, linfonodos, estadiamento do câncer, histopatologia.

\section{INTRODUÇÃO}

O câncer colorretal (CCR) é a quarta neoplasia mais incidente no Brasil ${ }^{1}$, a terceira causa de óbito por tumores malignos em mulheres e a quinta em homens ${ }^{2}$. Esses valores correspondem a um risco estimado de 12 casos novos para cada 100 mil homens e 15 para cada 100 mil mulheres ${ }^{3}$.

O prognóstico desse tipo de câncer pode ser considerado de moderado a bom, sendo o segundo tipo de câncer mais prevalente no mundo depois do câncer de mama, com uma estimativa de 2,4 milhões de casos diagnosticados nos últimos cinco anos com sobrevida média mundial de $44 \%{ }^{3}$.

A mortalidade persistiu inalterada nos últimos 50 anos a despeito dos avanços obtidos em diagnóstico e tratamento. Recentes avanços em técnicas e tratamento adjuvante têm resultado em um impacto modesto ou inexistente em pacientes com doença avançada ${ }^{4,5}$.

O estadiamento anátomo-patológico da peça operatória representa a principal ferramenta a ser utilizada isoladamente para inferir sobre o prognóstico e sobrevida de doentes com CCR ${ }^{6,7,8,9,10}$. Diferenças 
entre as populações no momento do diagnóstico e o comportamento biológico do tumor podem explicar as diferenças nas razões de incidência e mortalidade ${ }^{8}$.

Eventos clínicos e macroscópicos merecem ser contemplados em classificações prognósticas, pois quando aliados ao procedimento cirúrgico curativo e aos subsídios fornecidos pela microscopia clássica, podem melhor individualizar subgrupos de doentes com parâmetros semelhantes de sobrevida ${ }^{9}$.

O estádio clínico-patológico revelou ser a variável dominante independente que mais afeta a sobrevivência ${ }^{6,7,8,9,10,11}$, e poucos fatores foram identificados como tendo valor prognóstico independente do estadiamento de Dukes, tais como o grau de diferenciação celular e a invasão angiolinfática ${ }^{12,13}$. Contudo, a localização do tumor e a profundidade da invasão da parede intestinal também têm sido reportados como fatores prognósticos ${ }^{14}$.

Inicialmente, o estadiamento patológico pósoperatório foi usado tanto para a predição do prognóstico quanto para a indicação de terapia adjuvante ${ }^{15}$, mas um refinamento desse método pode levar a uma seleção mais justa de pacientes em triagem para esse tipo de terapia ${ }^{9,11}$.

O Sistema de Estadiamento Clínico-Patológico TNM é a diretriz atual do American Joint Committee ${ }^{5,15}$, e fornece informações para o estadiamento tanto do câncer retal quanto do câncer de cólon ${ }^{16}$.

Faz-se nescessário, dessa forma, complementar o sistema TNM clássico com outras variáveis prognósticas clínico-histológicas que permitam tomada de decisões mais fundamentadas a respeito da indicação de quimioterapia ${ }^{5}$.

Em 1940, Dukes demonstrou uma relação direta entre a extensão local do tumor e a incidência de metástases linfonodais ${ }^{11,13,17}$. Modificada por Astler e Coller em 1954, esse sistema de estadiamento clínicopatológico tem sido rotineiramente utilizado na prática clínica, já que muitos estudos demonstraram que 10 a $20 \%$ dos tumores limitados à submucosa e muscular própria já apresentam metástases linfonodais ${ }^{13}$.

A profundidade da invasão local, ou seja, o grau de penetração tumoral através da parede intestinal têm sido identificado como um fator prognóstico ${ }^{13,14,18}$ independente de sobrevida ${ }^{13,18}$, e associa-se a um maior risco de recidiva tumoral ${ }^{5}$. Segundo a classificação TNM e de Dukes, a sobrevida é de 91 a $99 \%$ para tumores que não comprometem além da muscular pró- pria, de 77 a $87 \%$ para aqueles além desta, e de 53 a $73 \%$ para os com metástase ganglionar ${ }^{5}$.

A graduação dos tumores segundo Dukes e Astler-Coller refere-se ao grau de invasão tumoral a partir da mucosa, atingindo paulatinamente, a submucosa, muscular, serosa, os tecidos perirretais, os gânglios linfáticos regionais e de cadeia, os órgãos vizinhos e distantes ${ }^{17}$.

Carcinomas invasivos geralmente se disseminam através da parede intestinal, indo em direção à submucosa, de onde drenam para os linfonodos epicólicos e desses para os paracólicos, eventualmente infiltrados por células tumorais ${ }^{13}$.

Assim, no que se refere à extensão do tumor na parede do órgão, quanto maior for sua penetração no cólon, menores serão as taxas de sobrevida ${ }^{11,13,18}$. O prognóstico é pobre quando o tumor se estende além da muscular própria, sendo que estes pacientes deveriam ser considerados de risco para o desenvolvimento de recorrência local, especialmente quando têm comprometimento linfonodal associado ${ }^{11}$.

Prova disso é que os tumores precoces, in situ ou localizados na muscular sem disseminação local, regional ou à distância, apresentam inclusive taxas de cura consideráveis ${ }^{18}$.

Até agora a profundidade da invasão da parede intestinal tem sido reportada como um fator prognóstico no $\mathrm{CCR}^{14}$ que, quando associada ao caráter de margem invasiva no câncer de reto, configura um significativo preditor de sobrevivência no estadiamento de Dukes e Jass ${ }^{18}$.

Já no que se refere ao grau de diferenciação tumoral, existe uma clara associação entre esse e o estádio do tumor no momento da ressecção ${ }^{12}$. O estádio tumoral e os tumores pouco diferenciados estão associados, sendo esses tumores mais avançados na ocasião da ressecção do que os tumores bem diferenciados ${ }^{8,12}$.

Tumores indiferenciados e pouco diferenciados foram associados a uma maior extensão local, invasão de vasos e linfonodos, além de maior risco de recidiva e metástases hepáticas ${ }^{19}$, atribuindo-se a esses um prognóstico mais reservado ${ }^{1,6,20}$.

Por conseguinte, quanto mais indiferenciado for o tumor, pior será seu prognóstico 1,6,17,10,21. Mesmo porque, tumores bem diferenciados respondem melhor à terapêutica adjuvante e têm velocidade de crescimento mais lenta, podendo ser detectados ainda em fases precoces ${ }^{22}$. 
Sob o ponto de vista anatômico, os adenocarcinomas podem apresentar-se como tumores mucosos ou mucíparos que se diferenciam dos demais por, eventualmente, apresentarem células em "anel de sinete" secretando mucina em abundância ${ }^{17}$.

Carcinomas mucinosos representam de 8 a $20 \%$ de todos os $\mathrm{CCR}^{23}$, e uma análise retrospectiva realizada no Oxford Cancer Registry ${ }^{7}$ entre 1995-2003 demonstrou que $10 \%$ dos tumores de cólon e 5\% dos tumores de reto são mucinosos.

$\mathrm{O}$ adenocarcinoma mucinoso, em qualquer grau de diferenciação, é considerado como fator localmente agressivo e de pior prognóstico ${ }^{9,10}$. Tumores mucinosos são os que, com mais assiduidade, se associam a um maior envolvimento da circunferência intestinal $-71 \%$ contra $33 \%$ dos não mucinosos ${ }^{9}$. Além disso, muitos estudos o associam a estádios mais avançados da doença ${ }^{19}$. Diversas razões são apontadas para a maior agressividade desses tumores, destacando-se a maior capacidade de invasão linfonodal, maior disseminação peritonial e maior incidência de recidiva local pélvica ${ }^{19}$.

A maioria dos pacientes jovens apresenta estadiamento avançado, tanto pelo sistema TNM como pela classificação de Dukes ${ }^{1,20}$. Atribui-se esse achado a uma maior prevalência de tumores mucinosos ou a um menor grau de diferenciação celular nessa população ${ }^{6}$.

As metástases ganglionares são um outro fator importante a ser avaliado na peça de ressecção do CCR. Sua importância vem do fato de que a linfadenectomia associada à ressecção do CCR é reconhecida desde 1908, quando Miles demonstrou que há uma tendência à recidiva tumoral na região de drenagem linfática do reto ${ }^{23}$.

Dukes por sua vez, concluiu que as metástases ganglionares representam o fator prognóstico de maior importância no que diz respeito à recorrência e sobrevida no CCR ${ }^{2,23}$. Quando presentes após terapia neoadjuvante, são ainda o maior fator prognóstico no câncer de reto, superadas apenas pela margem de ressecção circunferencial ${ }^{10,15}$.

Desde então, a pesquisa de linfonodos comprometidos é considerada o procedimento de maior relevância na análise anátomo-patológica de espécimes operatórios, em pacientes submetidos ao tratamento do CCR ${ }^{19}$, já que o envolvimento linfonodal é incorporado sem estadiamento clínico-patológico como uma variável independente no prognóstico do CCR ${ }^{2,5,11}$.
O número de linfonodos comprometidos na peça operatória é considerado um importante parâmetro para a avaliação da radicalidade da ressecção cirúrgi$\mathrm{ca}^{2,19}$. Alguns estudos demonstraram que a presença de micrometástases linfonodais, em pacientes com CCR, não influenciam o prognóstico, mas que o envolvimento de quatro ou mais linfonodos em pacientes estratificados na classe B de Dukes é um indicador de recorrência da doença ${ }^{24}$.

Dentre todos os parâmetros clínico-patológicos relacionados ao prognóstico do CCR, a determinação histológica da profundidade de invasão tumoral e a presença de metástases linfonodais ${ }^{2,18,24}$ são os mais importantes ${ }^{24}$, seguidos do grau de diferenciação celular ${ }^{19}$ e da secreção de mucina ${ }^{10}$.

A busca por fatores prognósticos que interfiram na sobrevida do CCR, tem sido uma preocupação constante da comunidade científica que estuda essa linha de pesquisa, uma vez que a sobrevida recebe influência de diversos fatores, os quais associados ou independentes, regulam o tempo de vida, não existindo regras de previsão, apenas evidências clínicas que geralmente podem ser alteradas ou modificadas individualmente ${ }^{18}$.

Tendo em vista todos esses aspectos, esse trabalho se propõe a realizar uma análise descritiva simples das prevalências desses quatro fatores de mau prognóstico presentes na peça operatória, correlacionando-os com a idade do paciente, número de linfonodos ressecados e positivos, e tamanho do tumor .

\section{MÉTODO}

Foram selecionados, em estudo transversal descritivo, os laudos histopatológicos de 144 pacientes submetidos ao tratamento cirúrgico, eletivo ou não, do CCR, com diagnóstico clínico-patológico prévio de adenocarcinoma colorretal, operados no Hospital Nossa Senhora da Conceição no Município de Tubarão SC, pelo pesquisador principal, no período compreendido entre janeiro de 1997 e dezembro de 2007.

Foram excluídos do estudo, todos os pacientes que preencheram os critérios abaixo:

1. Diagnóstico de CCR associado à Doença Inflamatória Intestinal (Doença de Chron ou Retocolite Ulcerativa).

2. Neoplasias reportadas como não condizentes com o tipo histológico de adenocarcinoma. 

adjuvante.

3. Pacientes submetidos à terapia neo-

Todos os pacientes foram operados pelo pesquisador principal, por laparotomia, e o tratamento cirúrgico instituído obedeceu ao padrão convencional de ressecções, incluindo linfadenectomia e no caso de tumores retais, a ressecção mesorretal.

As peças operatórias foram enviadas ao laboratório de patologia, sendo posteriormente laudadas de acordo com o Manual de Padronização de Laudos Histopatológicos da Sociedade Brasileira de Patologia 25 .

Os dados colhidos foram inseridos em um protocolo de pesquisa, que incluiu as variáveis de interesse para posterior análise estatística no SPSS 8.0.

Foi realizada, uma análise descritiva simples das prevalências na peça operatória do CCR dos quatro fatores de mau prognóstico de interesse no estudo e estabeleceram-se as associações descritas anteriormente.

\section{RESULTADOS}

Do total de 144 pacientes operados, 83 eram do sexo feminino (56.9\%). A média de idade foi de 61,21 anos (DP 14,81) e 12 pacientes tinham 40 anos ou menos $(8,3 \%)$.
No grupo de pacientes com menos de 40 anos não foram detectados estádios mais avançados da doença com relação ao $\mathrm{T}$, ao $\mathrm{N}$ ou ao grau de diferenciação/ presença de mucina ( $p=0,73, p=0,34$ e $p=0,22$ respectivamente).

Foram dispostos em um gráfico as prevalências dos quatro fatores de mau prognóstico de interesse no estudo (Gráfico 1).

Quanto ao grau de diferenciação, 76 pacientes apresentaram adenocarcinoma bem diferenciado $(52,8 \%), 56$ moderadamente diferenciados $(38,9 \%), 6$ pouco diferenciados $(4,2 \%)$ e nos 6 pacientes restantes foi observado adenocarcinoma mucinoso $(4,2 \%)$.

Estabeleceu-se a relação entre o grau de diferenciação celular/presença de mucina e o estadiamento $\mathrm{T}$ e $\mathrm{N}$, de forma que tumores crescentemente indiferenciados e secretores de mucina mostraram guardar relação positiva com o estadiamento $\mathrm{N}$, ao contrário do estadiamento $\mathrm{T}$, no qual essa relação não mostrou ser estatisticamente significativa (Tabela 1).

A média de gânglios dissecados foi de 11,17 (DP 0,59) e a média de gânglios positivos para neoplasia foi de 2,79 (DP 0,43).

O coeficiente de correlação de Pearson entre as variáveis "número de gânglios dissecados" e "número de gânglios positivos" foi de 0,460 ( $p<0,001)$. De modo que a ressecção de mais linfonodos aumenta a

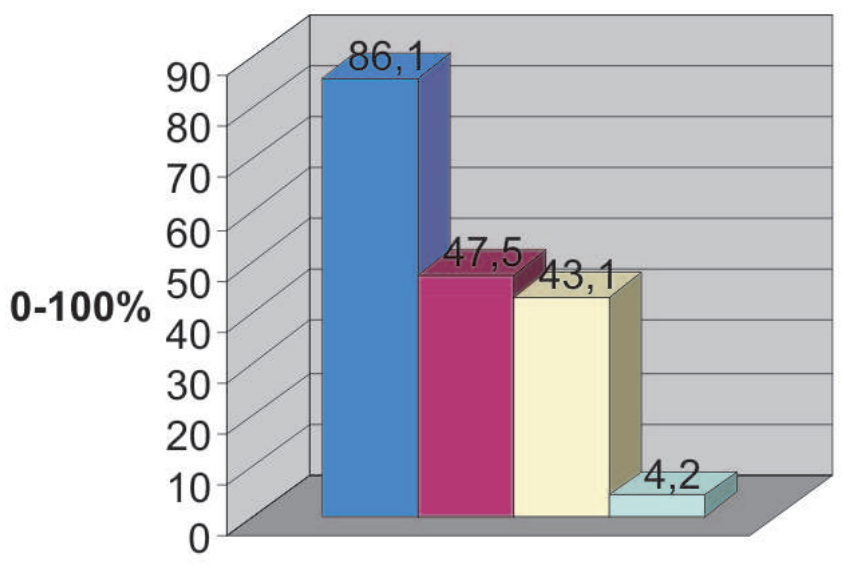

\begin{tabular}{|l|}
\hline \\
Tumores em estádio \\
T3/T4 de infiltração na \\
mucosa colônica \\
Presença de \\
metástase ganglionar \\
$\square$ Tumores \\
Indiferenciados \\
$\square$ Tumores Mucinosos
\end{tabular}

Gráfico 1 - Prevalência de quatro fatores de mau prognóstico nas peças operatórias de 144 pacientes submetidos a tratamento cirúrgico do câncer colorretal no Hospital Nossa Senhora da Conceição - Tubarão, SC. 
Tabela 1 - Distribuição do grau de diferenciação em relação ao Estadiamento $T$ e $N$ Patológico na peça operatória de pacientes submetidos ao tratamento cirúrgico do câncer colorretal no Hospital Nossa Senhora da Conceição - Tubarão, SC-1997-2007.

\begin{tabular}{|c|c|c|c|c|c|}
\hline Bem & renciado $(\%)$ & Moderadamente & Pouco & $\operatorname{Mucinoso}(\%)$ & Total(N) \\
\hline $\begin{array}{l}\text { Estadiamento } \\
\mathrm{T}^{*}\end{array}$ & & & & & \\
\hline Tis & 75 & - & 25 & - & 4 \\
\hline $\mathrm{T} 1$ & 66,7 & 33,3 & - & - & 3 \\
\hline $\mathrm{T} 2$ & 46,2 & 46,2 & - & 7,7 & 13 \\
\hline T3 & 54,5 & 40,2 & 2,7 & 2,7 & 112 \\
\hline $\mathrm{T} 4$ & 33,3 & 33,3 & 16,7 & 16,7 & 12 \\
\hline $\mathrm{N}^{* *}$ & & & & & \\
\hline No & 51,9 & 45,5 & 1,3 & 1,3 & 77 \\
\hline N1 & 64,9 & 24,3 & 2,7 & 8,1 & 37 \\
\hline $\mathrm{N} 2$ & 41,4 & 37,9 & 13,8 & 6,9 & 29 \\
\hline N3 & - & 100 & - & - & 1 \\
\hline
\end{tabular}

$* p=0,085$.

$* * p=0,037$.

chance de se encontrar um gânglio neoplásico (Tabela 2).

Quanto ao estadiamento T, 4 pacientes (2,8\%), eram portadores de adenocarcinoma in situ (Tis), 3 pacientes $(2,1 \%)$ foram classificados como T1, $13(9 \%)$ como T2, $112(77,8 \%)$ como T3 e 12 (8.3\%) como T4.

No que diz respeito à presença e extensão de metástase ganglionar, 77 pacientes $(53,5 \%)$ foram estadiados em N0, 37 (25.7\%) em N1, $29(20,1 \%) \mathrm{em}$ $\mathrm{N} 2$ e apenas 1 paciente $(0.7 \%)$ como N3.

Correlacionou-se então a média de gânglios dissecados/positivos com o $\mathrm{T}$, o $\mathrm{N}$ e o grau de diferen- ciação celular do tumor. Quanto maior o número de linfonodos dissecados, mais avançado foi o estadiamento T e N. Bem como quanto maior o número de gânglios positivos encontrados, mais avançados foram o estadiamento $\mathrm{T}$ e $\mathrm{N}$ patológicos e o grau de indiferenciação celular/ocorrência de adenocarcinoma mucinoso (Tabela 3).

Com relação ao tamanho do tumor, foram classificados como pequenos tumores cujo maior diâmetro foi menor ou igual a $35 \mathrm{~mm}$ e como grandes tumores cujo maior diâmetro foi superior a $35 \mathrm{~mm}$. Dezenove tumores foram classificados como pequenos $(13,2 \%)$

Tabela 2 - Proporção de linfonodos positivos de acordo com o número de gânglios dissecados em pacientes submetidos à ressecção cirúrgica do câncer colorretal no Hospital Nossa Senhora da Conceição Tubarão, SC-1997-2007.

\begin{tabular}{lcc}
\hline Número de pacientes & Média de Gânglios Dissecados & Média de Gânglios Positivos \\
\hline 25 & $0-4$ & 0,72 (DP 1,10) \\
38 & $5-9$ & 1,50 (DP 2,15) \\
44 & $10-14$ & $2,31 \quad(\mathrm{DP} 3,58)$ \\
21 & $15-19$ & 5,38 (DP 5,93) \\
8 & $20-24$ & $0,50 \quad(\mathrm{DP} 1,06)$ \\
2 & $25-29$ & 19,50 (DP 2,12) \\
6 & $>$ ou $=30$ & $11,66(\mathrm{DP} 14,20)$ \\
\hline
\end{tabular}

$p_{\text {Kruskal-Wallis }}=0,0109$. 
Tabela 3 - Relação entre o Estadiamento T, $N$ e Grau de Diferenciação Celular com a Média de Gânglios Dissecados e de Gânglios Positivos na peça operatória de pacientes submetidos ao tratamento cirúrgico do câncer colorretal no Hospital Nossa Senhora da Conceição, 1997-2007.

\begin{tabular}{|c|c|c|c|}
\hline & Médi & a de Gânglios dissecados & Média de Gânglios positivos \\
\hline \multirow[t]{5}{*}{$\mathrm{T}^{*}$} & Tis & $5 \quad(\mathrm{DP} 4,29)$ & (DP 0) \\
\hline & $\mathrm{T} 1$ & $5,30($ DP 6,11$)$ & (DP 0) \\
\hline & & 8,69 (DP 4,38) & $0,69 \quad(\mathrm{DP} 1,49)$ \\
\hline & & $11,46(\mathrm{DP} 7,25)$ & 2,92 (DP 5,01) \\
\hline & $\mathrm{T} 4$ & 14,66 (DP 6,37) & $5,58 \quad(\mathrm{DP} 8,79)$ \\
\hline \multicolumn{4}{|l|}{$\mathrm{N}^{* *}$} \\
\hline & No & $10,71(\mathrm{DP} 6,95)$ & $(\mathrm{DP} 0)$ \\
\hline & & $8,59(\mathrm{DP} 5,00)$ & $2,13 \quad(\mathrm{DP} 1,39)$ \\
\hline & & $15,69(\mathrm{DP} 7,80)$ & $10,79 \quad(\mathrm{DP} 6,71)$ \\
\hline & N3 & $11,00(\mathrm{DP} 0)$ & $11,00 \quad$ (DP 0) \\
\hline \multicolumn{4}{|c|}{ Grau de Diferenciação Celular*** } \\
\hline Bem-diferenciado & & $10,65(\mathrm{DP} 7,41)$ & $2,57 \quad($ DP 5,15$)$ \\
\hline Moderadamente diferenciado & & $11,33(\mathrm{DP} 6,17)$ & $2,00 \quad(\mathrm{DP} 3,58)$ \\
\hline \multicolumn{4}{|l|}{ Pouco } \\
\hline diferenciado & & $12,66(\mathrm{DP} 10,38)$ & $10,16$ (DP 10,42$)$ \\
\hline Mucinoso & & $14,66(\mathrm{DP} 7,33)$ & $5,66 \quad($ DP 6,59$)$ \\
\hline
\end{tabular}

Valor de p referente à média de gânglios dissecados:

$* p_{\text {Kruskal-Wallis }}=0,017$.

$* * p_{s}=0,00046$.

$* * * p_{\text {Kruskal-Wallis }}=0,43$.

Valor de p referente à média de gânglios positivos:

$* p_{\text {Kruskal-Wallis }}=0,036$.

$* * p<0,000001$.

$* * * p_{\text {Kruskal-Wallis }}=0,001$.

e 125 como grandes $(86,8 \%)$. O tamanho do tumor se correlacionou positivamente com estádio T mais avançado $(p=0,00000790)$.

\section{DISCUSSÃO}

O CCR é uma das causas mais freqüentes de morte no mundo desenvolvido e a sobrevida depende claramente do estádio ${ }^{6,7,8,9,10,26}$, mas há muitos fatores que podem ajudar a prever o prognóstico ${ }^{26}$.

A média de idade encontrada nesse estudo foi de 61 anos, próxima à média de idade encontrada em outras séries ${ }^{1,5,6,8,9,24,27,28}$, sendo o risco desse câncer tanto maior quanto maior a faixa etária, uma vez que mais de 50\% dos casos ocorrem em indivíduos com mais de 50 anos ${ }^{29}$.

A idade por si só não constitui fator de risco de mortalidade operatória ou de maior tempo de sobrevida,

exercendo essa influência o estado fisiológico do paciente ${ }^{9,29}$, embora as complicações pós-operatórias sejam mais freqüentes e graves nos doentes com mais de 65 anos ${ }^{30}$.

Em nosso estudo, o percentual de pacientes jovens foi ligeiramente superior àquele encontrado em outras séries ${ }^{1,6,30,31}$ e esse grupo não apresentou estadiamento $\mathrm{T}$ e $\mathrm{N}$ mais avançado ou menor grau de diferenciação celular, embora, em alguns estudos ${ }^{1,31}$, a proporção de tumores retais pobremente diferenciados tenha sido maior em pacientes com menos de 30 anos.

Oitenta e três pacientes eram do sexo feminino $(56,9 \%)$, em consonância com o encontrado na maioria dos artigos revisados ${ }^{2,9,17,23}$, e contrariando outras séries ${ }^{27,32}$ nas quais não houve predomínio discreto do CCR nesse sexo, embora tenha sido descrito um aumento de $69 \%$ no crescimento médio anual dos casos de CCR para homens e de $60 \%$ para mulheres ${ }^{29}$.

Tumores de diâmetro superior a $35 \mathrm{~mm}$ em nossa casuística apresentaram-se com estádio T mais avançado e esse fato desperta grande interesse no que 
diz repeito à classificação TNM que, no geral, não atribui valor de importância ao tamanho da neoplasia ${ }^{16}$.

Yasuda et al ${ }^{24}$, contudo, não encontraram relação significativa entre a recorrência do CCR e a presença de tumores de diâmetro superior a $35 \mathrm{~mm}$.

Sabe-se que a invasão venosa mostrou reduzir significativamente a sobrevida independentemente do estádio ou grau do tumor ${ }^{23}$ e Folkman, em 1971, demonstrou que o crescimento tumoral é considerado angiogênese-dependente $23,18,33$.

À medida que o tumor cresce no envolvimento circular do intestino, o número de metástases linfonodais aumenta, a recidiva é mais freqüente e a sobrevida diminui. Na prática, tumores de crescimento intramural, mesmo quando pequenos, estariam associados a um pior prognóstico ${ }^{9}$.

Ainda assim, dentre o amplo espectro de características desfavoráveis do paciente com CCR, destaca-se seu caráter de grande imprevisibilidade quanto ao prognóstico do paciente ${ }^{34}$, e é grande a demanda de outros parâmetros que ampliem o percentual prognóstico desses doentes, dentre os quais estão o tamanho do tumor ${ }^{9}$.

Já o estadiamento de Dukes foi detectado como um fator prognóstico ${ }^{15}$, embora não venha sendo utilizado como critério de triagem para terapia adjuvante ${ }^{12}$. Todavia, assim como em outras séries ${ }^{26,35,36}$, nesse estudo o estádio do CCR foi definido de acordo com a classificação TNM recente.

No que se refere ao estadiamento $\mathrm{T}$, a distribuição encontrada nesse estudo foi percentualmente próxima àquela encontrada na maioria dos artigos revisados $4,8,13,33,37$, nos quais a maior parte dos pacientes encontravam-se em estádio T3; embora saiba-se que o cuidado com o exame histopatológico determina os resultados do T patológico. Em geral, para tumores gastrointestinais, o $\mathrm{T}$ patológico depende da profundidade de invasão ${ }^{35}$.

Além disso, o patologista deve fatiar cuidadosamente o tumor e selecionar, para o exame histopatológico, áreas suspeitas de invasão profunda. Isso porque tumores que invadem órgãos adjacentes por contiguidade, mesmo sem evidência de metástases à distância, devem ser submetidos à ressecção alargada, uma vez que a ressecção radical não parece influenciar o prognóstico ${ }^{38}$.

Sabe-se também que, embora a presença de doença tumoral extra intestinal reduza a sobrevida, a invasão direta do tumor a estruturas adjacentes não contra-indica a ressecção de metástases hepáticas no CCR ${ }^{39}$.

A determinação histológica da profundidade de invasão tumoral e o grau de invasão tumoral são alguns dos parâmetros histopatológicos mais importantes relacionados ao prognóstico do $\mathrm{CCR}^{24}$, embora a extensão da infiltração serosa e a invasão venosa por células cancerosas tenham sido detectadas como fatores de mau prognóstico no câncer de reto e não de cólon ${ }^{15}$.

A relevância prognóstica do tumor residual e da classificação TNM em pacientes com CCR, demonstrou que o $\mathrm{T}$ e o $\mathrm{N}$ não influenciam o prognóstico de pacientes com tumor residual ${ }^{40}$.

Conforme o esperado, o estadiamento $\mathrm{N}$ nesse estudo aproximou-se do encontrado em grande parte das séries estudadas 2,7,18,23,24,41, nas quais a maior parte dos pacientes foram incluídos no estadio No.

O envolvimento de linfonodos regionais é de crítica importância para o prognóstico ${ }^{24,42,43}$ e sua análise é mandatória ${ }^{24}$, mas o acesso macroscópico dos linfonodos é inviável, já que uma considerável parte das metástases linfonodais ( mais de 30\%) têm em seu maior diâmetro $3 \mathrm{~mm}$ ou menos ${ }^{24,43}$.

A recomendação quanto ao número de linfonodos que devem ser ressecados durante a cirurgia ainda é fonte de bastante controvérsia. Conforme nosso estudo, a média de gânglios dissecados foi de 11, e desses, em média, 2,8 apresentavam comprometimento neoplásico.

Wong et $\mathrm{al}^{44}$, em sua pesquisa, obtiveram uma média de 17 gânglios ressecados observados na peça operatória do CCR, e 38,6\% dos pacientes tinham pelo menos 1 nodo positivo, sugerindo, em conformidade com nosso estudo, que quanto maior o número de gânglios dissecados maior a probabilidade de encontrarmos linfonodos positivos.

Embora o maior número de linfonodos examinados seja aceito como fator prognóstico positivo, esse benefício é atribuído por Jass et al. a uma ressecção cirúrgica mais efetiva nessa condição e não a uma melhor resposta imunológica contra a neoplasia ${ }^{24}$.

Lanza et al ${ }^{36}$ demonstraram que a análise de um pequeno número de linfonodos regionais pode resultar em um subestadiamento de tumores classificados como $\mathrm{N} 0$ e gânglios maiores que $3 \mathrm{~mm}$ de diâmetro deveriam ser considerados metastáticos pela classificação TNM, razão pela qual alguns pacientes sem 
metástases linfonodais têm recorrência pós-operatória ou desenvolvem metástases ${ }^{24}$.

Já Swanson et al ${ }^{23,24}$ concluem que um mínimo de 13 linfonodos devem ser examinados para que um tumor T3 seja considerado negativo para metástases, e Cserni et al ${ }^{43}$ sugerem que para tumores T3N0M0 não existe um limite específico de linfonodos necessários e que a linfadenectomia deve ser tão ampla quanto possível ${ }^{23,24}$.

Cianchi et al ${ }^{37}$ ao recordar a importância da estratificação prognóstica de pacientes Dukes B sugerem que portadores de oito ou mais linfonodos positivos têm sobrevida semelhante aos pacientes Dukes C, propondo a elaboração de um novo modelo de prognóstico para pacientes Dukes B que poderiam se beneficiar da terapia adjuvante ${ }^{26,43}$.

A probabilidade de encontrarmos nodos positivos é maior quando mais de 10 gânglios forem dissecados e quando o adenocarcinoma for indiferenciado ${ }^{45}$. Beneveneto et al ${ }^{20}$ sugerem a ressecção de uma média de 20 linfonodos.

Metástases linfonodais são consideradas fator independente de prognóstico ${ }^{2,19,46}$ e a extensão da infiltração tumoral nos linfonodos tem um impacto negativo na sobrevida livre da doença em pacientes com câncer retal ${ }^{46}$.

Em estudos prévios, a sobrevida em 5 anos caiu significativamente quando o número de linfonodos positivos foi maior que 3 e o nível de metástases linfonodais foi fortemente correlacionado com a sobrevivência em 5 anos: $72-85 \%$ para N1, 51-67\% e para N2 e $30-56 \%$ para N3 ${ }^{24}$.

Broll et al. demonstraram que não há diferença significativa na sobrevida livre da doença entre pacientes com micrometástases linfonodais negativas e positivas no estádio I/II do $\mathrm{CCR}^{24} \mathrm{e}$ a invasão linfática mostrou não ter efeito na sobrevivência em baixos estádios, apesar de ter efeito negativo significante quando presente em altos estádios ${ }^{26}$.

Metástases linfonodais são o fator prognóstico mais importante no CCR, de forma que a investigação patológica, além da avaliação histológica de rotina de linfonodos, pode identificar maior número de pacientes com alto risco de recorrência ${ }^{28}$.

A distribuição do grau de diferenciação celular nas peças operatórias dos pacientes desse estudo também foi semelhante à encontrada na literatura revisada ${ }^{6,7,9,12,41}$, na qual a maior parte dos pacientes exibiu adenocarcinoma bem diferenciado.
O grau de diferenciação celular é um importante fator na determinação do prognóstico no CCR 1,6,20. A graduação histológica mostrou ter efeito significativo na sobrevivência independentemente do estadiamento, mas seu valor prognóstico pode ser melhorado se critérios mais objetivos forem desenvolvidos para melhor avaliar essa variável ${ }^{16}$.

Broders classificou os adenocarcinomas retais em 4 tipos, em conformidade com o percentual de células malignas encontradas por campo no microscópio, mas esse constitui apenas um dos vários parâmetros importantes para avaliar o real grau de diferenciação tumoral ${ }^{17}$.

Sabe-se que o grau de diferenciação celular constitui a variável histológica mais freqüentemente utilizada em associação ao estadiamento anátomo-patológico ${ }^{1,20}$. Embora em nosso estudo pacientes jovens não tenham apresentado tumores mais indiferenciados, essa é uma e, às vezes, a única variável de agressividade biológica mais prevalente nos pacientes jovens ${ }^{1,6,20}$.

O tipo histológico do tumor e a indiferenciação afetam a sobrevivência em altos e baixos estádios, mas em alguns trabalhos ${ }^{26}$ essa diferença não foi significativa; embora Müssnich et al ${ }^{47}$ tenham demonstrado que a diferenciação tumoral tenha efeito prognóstico independente na predição da sobrevida de pacientes com câncer de reto.

Jass et al ${ }^{23}$ procederam a uma análise multivariada de sete variáveis relacionadas à diferenciação tumoral no câncer de reto que, quando ajustadas à classificação de Dukes, demonstraram que somente a infiltração linfocítica foi um preditor independente do prognóstico.

Recente estudo ${ }^{8}$ demonstrou que tumores do lado direito do cólon foram significativamente menos diferenciados que os do lado esquerdo, e se sustenta a hipótese de que tumores do lado direito e esquerdo do cólon diferem em sua etiologia.

Por fim, dentre os aspectos tumorais mais confiáveis na predição do prognóstico, a presença de mucina obteve um dos maiores índices de correlação com a sobrevivência ${ }^{19,23,34}$, enquanto que em nosso estudo essa foi associada a um estadiamento $\mathrm{N}$ mais avançado.

Farhoud et al ${ }^{9}$ relatam que os tumores mucinosos são os que, com mais assiduidade se associam a um maior envolvimento da circunferência intestinal $-71 \%$ contra $33 \%$ dos não mucinosos. 
Rev bras Coloproct Outubro/Dezembro, 2008
Fatores de Mau Prognóstico nas Pecas Operatórias de Pacientes Submetidos ao Tratamento Cirúrgico do Câncer Colorretal Marília Granzotto Volpato e Cols.
Vol. 28
Contudo, recente estudo ${ }^{48}$ demonstrou que, embora o adenocarcinoma com células em anel de sinete antecipe um pior prognóstico, a sobrevida de pacientes com tumores secretores de mucina foi semelhante àquela encontrada nos outros tipos de adenocarcinoma.

\section{CONCLUSÃO}

Pacientes jovens não apresentaram estadiamento $\mathrm{T} / \mathrm{N}$ mais avançado, bem como menor grau de diferenciação celular e presença de adenocarcinoma mucinoso quando comparados aos pacientes com mais de 40 anos.

$\mathrm{O}$ avanço do estadiamento $\mathrm{N}$ relacionou-se positivamente com a ocorrência de maior número de casos de adenocarcinomas pouco diferenciados e secretores de mucina.
Tumores grandes obtiveram estádio $\mathrm{T}$ mais avançado, sugerindo que tumores com diâmetro maior que $35 \mathrm{~mm}$ atuem como fator de mau prognóstico.

Quanto maior o número de linfonodos ressecados, maior é a possibilidade de encontrar gânglios positivos; mais avançados são o $\mathrm{T} / \mathrm{N}$ patológicos e menor o grau de diferenciação celular, bem como maior é a ocorrência de adenocarcinoma mucinoso; de forma que uma linfadenectomia inadequada pode produzir um subestadiamento do CCR.

\section{AGRADECIMENTOS}

Os autores agradecem a Thiago Mamoru Sakae, MsC; pelo auxílio na análise estatística e a Ricardo Beckhauser Kuhnen pelo auxílio na coleta dos dados utilizados neste artigo.

\begin{abstract}
BACKGROUND: The pathologic staging of the surgical peace represents the leading method of measuring the prognostic and survival of patients with colorectal cancer (CRC). AIM: To determine the prevalence of T, N, cellular differentiation, and the presence of mucus, on the surgical peaces of patients submitted to surgical treatment of CRC. METHODS: The histopathology results of 144 patients were evaluated in regard to their clinical-pathology staging. RESULTS: The mean-age found was 61.21 years. Twelve patients aged $<40$ years $(8.3 \%)$. One hundred and twenty five patients $(\mathbf{8 6 , 8 \%})$ showed large tumors (>35mm). Twelve hundred patients $(\mathbf{7 7 , 8 \%})$ were found in T3 stage, and 77 patients $(53,5 \%)$ were ranked as NO. Seventy six patients $(52,8 \%)$ showed carcinoma with high differentiation. Six patients $(4.2 \%)$ had mucinous carcinoma. The mean parches of lymph nodes realized was 11.1 and the positive lymph nodes had a mean of 2.79. CONCLUSION: Young patients did not have more advanced $\mathbf{T}$ and $\mathbf{N}$ staging or higher mucus secretion and cellular indifferentiation. Large tumors obtained more advanced $\mathrm{T}$ staging, and the T/N also had a positive relationship with a higher mean of lymph nodes parched. The retreat of a large number of lymph nodes increased the chances of finding a neoplasic lymph node and the finding of positive lymph node increased the $T /$ $\mathrm{N}$ staging, the cellular indifferentiation and the mucus presence.
\end{abstract}

Key words: Colorectal neoplasm, prognosis, lymph nodes, neoplasm staging, histopathology.

\section{REFERÊNCIAS}

1. Carneiro Neto JD, Barret JBP, Freitas NS, Queiroz MA. Câncer Coloretal: Características clínicas e anatomo-patológicas em pacientes com idade inferior a 40 anos. Rev Bras Coloproct 2006; 26(4): p 430-435.

2. Pereira Júnior T, Torres RAB, Nogueira AMMF. Acometimento metastático linfonodal no câncer colorretal. Arq Gastroenterol 2006; 43(2): p 89-93.

3. Ministério da Saúde. Secretaria Nacional de Assistência à Saúde. Instituto Nacional do Câncer. Estimativas da incidência e mortalidade por câncer no Brasil. Disponível em: <http://www.inca.gov.br/ estimativa2006/sintese.html>. Acesso em 28 ago 2007.

4. Almeida FFN, Araujo EA, Santos FPS, Franco CJCS, Santos VR, Nahas SC, et al. Colorectal Cancer Screening. Rev Hosp Clín Fac Med S Paulo 2000; 55(1): p 35-42.
5. Zúñiga AA, López KF, Boza C, Zúñiga DA, Rahmer A, Oyarzún IC et al. Análisis uni y multivariado de los factores pronósticos en cáncer de colon. Rev Chilena de Cirugía 2005; 57(2): p 143-148.

6. Lupinacci RM, Campos FG, Araújo SE, Imperiale AR, Seid VE, Habr-Gama A et al. Análise comparativa das características clínicas, anátomo-patológicas e sobrevida entre pacientes com câncer colo-retal abaixo e acima de 40 anos de idade. Rev Bras Coloproct 2003; 23(3): p155-162.

7. Green J, Watson J, Roche M, Beral V, Patnick J. Stage, grade and morphology of tumors of the colon and rectum recorded in the Oxford Cancer Registry, 1995-2003. Br J Cancer 2007; 96 (1): p 140-142.

8. Keating J, Pater P,Lolohea S, Wickremesekera K. The epidemiology of colorectal cancer: what can we learn from the New Zeland Cancer Registry?. N Z Med J 2003; 116 (1174): p 437. 
9. Farhoud S, Bromberg SH, Barreto E, Godoy AC. Variáveis clínicas e macroscópicas que influenciam o prognóstico do carcinoma colorretal. Arq Gastroenterol 2002; 39(3): p 163172.

10. Monteiro EP, Salem JB, Taglietti EM, Albuquerque IC, Formiga GJ. Neoplasia colorretal até os 40 anos - Experiência em cinco anos. Rev Bras Coloproct 2006; 26(2): p 156-161.

11. Chapuis PH, Dent OF, Fisher R, Newland RC, Pheils MT, Smyth, Eet al. A multivaiate analysis of clinical and pathological variables in prognosis after resection of large bowel cancer. $\mathrm{Br}$ J Surg 1985; 72(9): p 698-702.

12. Gagliardi G, Stepniewska KA, Hershman MJ, Hawley PR, Talbot IC. New Grade Related prognostic variable for rectal cancer. Br J Surg 1995; 82(5): p 599-602.

13. Benevento A, Boni L, Dionigi G, Carcano C, Capella G, Casula $\mathrm{G}$ et al. The mesenteric and antimesenteric location of colorectal cancer. Surgeon 2004; 2(4): p 214-220.

14. Tominaga T, Sakabe T, Koyama Y, Hamano K, Yasutomi M, Takahashi T, et al. Prognostic Factors for Patients with Colon or Rectal Carcinoma Trated with Resection Only - Five-year Follow-up Report. Cancer 1996; 78(3): p 403-408.

15. Nagtegaal ID, Marijnen CAM, Rutten HJ, Velde CJH, Krieken HJM. Combinations of tumor and tratment parameters are more discriminative for prognosis than the present TNM System in rectal cancer. J Clin Oncol 2007; 25(13): p 16471650.

16. Williams N S. Câncercolorretal: Epidemiologia, Etiologia, Patologia, Manifestações Clínicas e Diagnóstico. In: Keighley MRB, Williams NS. Cirurgia do Ânus, Reto e Colo. São Paulo: Ed.Manole, 1998 p.813-14.

17. Cruz GMG, Ferreira RMRS, Neves PM. Cancer Retal: Estudo demográfico, diagnóstico e estadiamento de 380 pacientes acompanhados ao longo de quatro décadas. Rev Bras Coloproct 2004; 24(3): p 208-224.

18. Cianchi F, Palomba A, Luca M, Boddi V, Asirelli G, Perigli G et al. Tumor angiogenesis in lymph node-negative rectal cancer: Correlation with clinicophatological parameters and prognosis. Ann Surg Oncol 2002; 9(1): p 20-6.

19. Henrrique-Filho C, Bromberg SH, Barreto E, Godoy AC, Mattosinho-França LC. Valor prognóstico do grau de diferenciação celular, da presença de muco e do padrão de crescimento da margem invasiva em adenocarcinomas colorretais Dukes B. Arq Gastroenterol 2004; 41(3): p 185-189.

20. Saad-Hossne R, Prado RG, Bakonyi Neto A, Lopes PS, Nascimento SM, Santos CRV et al. Estudo retrospectivo de pacientes portadores de câncer colorretal atendidos na Faculdade de Medicina de Botucatu no período de 200-2003. Rev Bras Coloproct 2005; 25(1): p 3137.

21. Compton CC, Fielding LP, Burgart LJ, Conley B, Cooper HS, Hamilton SR et al. Prognostic Factors in Colorectal Cancer College of American Consensus Statement 1999. Arch Pathol Lab Med 2000; 124(7): p 979-994.
22. Jucá MJ Sobrevida no Câncer de Cólon - Análise da influência da localização do tumor. Capítulo XXI. Disponível em: < http://www.proctosite com/. library/ books/livro_reis_novo/ cap21.pdf >.Acesso em 26 set 2007.

23. Pinho MSL, Ferreira LC, Kleinubing Júnior H, Fischer C, Pereira Filho AJG, Wengerkievicz AC. Câncer colorretal: Análise do valor prognóstico do número total de linfonodos identificados na peça de ressecção. Rev Bra Coloproct 2004; 24(2): p 126-130.

24. Yasuda K, Adachi Y, Shiraishi N, Yamagushi K, Hirabayashi Y, Kitano S. Pattern of lymph node micrometastasis and prognosis of patients with colorectal cancer. Ann Surg 2001; 8(4): p 300-304.

25. Manual de Padronização de Laudos Histopatológicos / Sociedade brasileira de Patologia;[editores: Carlos Eduardo Bacchi, Marcello Fabiano de Franco, Paulo Cirilo Cardoso de Almeida] - 3ed - São Paulo : Reichmann \& Autores Editores, 2005

26. Krebs B, Kozelj M, Kavalar R, Gajzer B, Gadzijev EM. Prognostic value of additional pathological variables for longterm survival after curative resection of rectal cancer. World $\mathrm{J}$ Gastroenterol 2006; 12(28): p 4565-8.

27. Weitz J, Kienle P, Lacroix J, Willeke F, Benner A, Lehnert T et al. Dissemination of Tumor Cells in Patients Undergoing Surgery for Colorecal Cancer. Clin Cancer Res 1998; 4(2): $p$ 343-348.

28. Iddings D, Ahmad A, Elashoff D, Bilchik A. The prognostic effect of micrometastases in previously staged lymph node negative (N0) colorectal carcinoma: a meta-analysis. Ann Surg Oncol 2006; 13(11): p1386-92.

29. Habr-Gama A. Câncer colorretal - A importância da prevenção. Arq gastroenterol 2005; 42(1): p 2-3.

30. Malheiros AP, Teixeira MG, Habr-Gama A, Alcântara PSM. Resultados do tratamento cirúrgico do Câncer Colorretal em doentes de idade até 64 anos e de 65 anos ou mais. Rev bras Coloproct 2005; 25(2): p128-136.

31. Morris M, Platell C, Iacopeta B. A population-based Study of Age-relates Variation in Clinicophatological Features, Molecular Markers and Outcome from Colorectal Cancer. Anticancer Res 2007; 27(4C): p 2833-2838.

32. Saad-Hossne R. Estudo retrospectivo de pacientes portadores de câncer colorretal atendidos na Faculdade de Medicina de Botucatu no período de 200-2003. Rev bras Coloproct 2005; 25(1): p 31-37.

33. Tarta C, Teixeira CR, Silva VD, Neto CC, Prolla JC, Gus P. Angiogênese no Carcinoma Colorretal - Revisão e Perspectivas. Rev bras Coloproct 2000;20(4): p 227-230.

34. Pinho MSL. Estadiamento Molecular do Câncer Colorretal: $\mathrm{O}$ futuro se aproxima. Rev bras Coloproct 2055; 25(3): p 279284.

35. Hermanek P. pTNM and residual tumor classifications: problems of assessment and prognostic significance. World J Surg 1995; 19(2): p 184-90. 
36. Lanza G, Gafà R, Decarli N. Pathological factors involved in lymph node status determination in colorectal carcinoma: analysis of 166 cases with long-term follow-up. Pathologica 2001; 93(6): p 631-639

37. Cianchi F, Messerini L, Comin CE, Boddi V, Perna F, Perigli G et al. Pathologic determinants of survival after resection of T3N0 (Stage IIA) colorectal cancer: proposal for a new prognostic model. Dis Colon Rectum 2007; 50(9): p 13321341.

38. Barbosa-Silva T, Carvalho EES, Campos JEGO, Da Silca RG, Da Conceição AS et al. Ressecção alargada em pacientes com câncer colorretal localmente invasivo. Rev bras Coloproct 2002; 22(1): p 27-32.

39. Chedid AD, Villwoch MM, Chedid MF, Rohde L. Fatores prognósticos na ressecção de metástases hepáticas de Câncer Colorretal. Arq Gastroenterol 2003; 40(3): p 159-165.

40. Hermanek P Jr, Wiebelt H, Riedl S, Staimmer D, Hermanek P. Long-term results of surgical therapy of colon cancer. Results of the Colorectal Cancer Study Group. Chirurg 1994; 65(4): p 287-97.

41. Kim J, Huynh R, Abraham I, Kim E, Kumar RR. Number of lymph nodes examined and its impact on colorectal cancer staging. Am Surg 2006 ; 72(10): p 902-905.

42. Jestin P, Påhlman L, Glimelius B, Gunnarsson U. Cancer staging and survival in colon cancer is dependent on the quality of the pathologists' specimen examination. Eur J Cancer 2005; 41(14): p 2071-1078.

43. Cserni $\mathrm{G}$. The influence of nodal size in the staging of colorecal carcinomas. J Clin Pathol 2002; 55(5): p 386-390.
44. Wong JH, Johnson S, Hemmings D, Hsu A, Imai T, Tominaga GT. Assesing the quality of colorectal cancer staging: documenting the process in improving the stage of node-negative colorectal cancer. Arch of Surg 2005; 140 (9): p 881-6.

45. Kuhnen RB, Koch KS. Número de gânglios dissecados em peças operatórias de pacientes submetidos à ressecção cirúrgica de câncer colorretal. Retrospectiva de 10 anos HNSC Tubarão - SC. Rev bras Coloproct 2007; 27(4): p 417-422.

46. Ptok H, Meyer F, Steinert R, Vieth M, Ridwelski K, Lippert $\mathrm{H}$ et al. No prognostic impact of isolated lymphovascular invasion after radical resection of rectal cancer-results of a multicenter observational study. Int J Colorectal Dis 2007; 22(7): p 749-756.

47. Müssnich HG; Moreira LF; Gus P; Pimentel M; Simon T; Santos MB. Fatores Prognósticos e Sobrevida no Adenocarcinoma Primário de Reto. Rev bras Coloproct 2008; 28(1): p 62-71.

48. Kang H, O'Connell JB, Maggard MA, Sack J, Ko CY. A 10Year Outcomes Evaluation of Mucinous and Signet-Ring Cell Carcinoma of the Colon and Rectum. Dis of The colon and rectum 2005; 48(6): p 87-89.

\section{Endereço para correspondência: KAISER DE SOUZA KOCH}

Av. Marcolino Martins Cabral, 2075 Ed. Interclínicas - $7^{\circ}$ andar 88075-001

Tubarão/SC

Tel.: (48) 3631-1500/3631-1560

E-mail:kkoch@unisul.br 